\title{
Perception of complex motion paths under three conditions of stimulation
}

\author{
ANN O'LEARY \\ Rutgers University, New Brunswick, New Jersey \\ MARGARET L. MCMAHON \\ Ursinus College, Collegeville, Pennsylvania \\ and \\ HANS WALLACH \\ Swarthmore College, Swarthmore, Pennsylvania
}

\begin{abstract}
In the experiment reported, the accuracy of reproductions of the shapes of more or less complex motion paths was used to examine the effectiveness of two stimulation conditions, of ocular pursuit, and of configurational change when the latter took the form of induced motion. Reproductions of the apparent motion paths of an objectively stationary spot set into perceived motion by its moving background were found to be as accurate as the reproductions of identically shaped paths perceived when the spot underwent real motion that was given by ocular pursuit. A third set of motion-path reproductions was obtained when two conditions of stimulation participated in mediating a perceived motion: The horizontal component of each motion was given as induced motion, and the vertical component, by ocular pursuit. The two processes evoked by the two different stimulation conditions were known to combine to form a single perceived motion. The results of this "combination" condition were compared with those of the single stimuli described above. In one case, the comparison was with the results of ocular pursuit, and in the other, it was with configurational change. Although perceiving combined motions was, for our subjects, a novel perceptual event, reproductions of the motion paths that were perceived under these conditions were, with the exception of one stimulus display, not worse than those made when the motions were given under the other two conditions.
\end{abstract}

When an object moves in a homogeneous field, its motion is perceived because the direction from which it is seen changes gradually; its motion is given as a subjectrelative displacement. This subject-relative displacement is mediated either by eye movements that track the moving object or by the displacement on the retina of the image of the moving object when the eyes rest on a stationary point. When the moving object is among stationary objects or is seen against a patterned background, it changes its location relative to these landmarks and its motion is also given by its displacement relative to them. That such object-relative displacement may indeed mediate motion perception can be shown by reversal of motion and immobility. When the object is at rest and the patterened background moves in such a way that the previous relative displacement is duplicated, most subjects will report, in addition to motion of the background, the same motion of the object as before, although that motion is no longer produced subject-relatively. The motion that is perceived under these conditions is called induced mo-

This work was supported by Grant 11089 from the National Institute of Mental Health to Swarthmore College, Hans Wallach, principal investigator. H. Wallach's mailing address is Department of Psychology, Swarthmore College, Swarthmore, PA 19081. tion. Object-relative displacements are given on the retina as a configurational change.

Induced motion is thus a direct manifestation of one of the stimulation conditions that mediate the perception of motion. Although this view was presented early on by Duncker (1929), it is not widely accepted, and alternative explanations of induced motion have been proposed (Post, 1986; Regan, 1986; Rock, 1983). In the present research, we attempted to determine whether the quality of perceived motion based on induced-motion conditions was as good as that of motion mediated by other stimulation. We presented a series of more or less complex motion paths that were perceived under three different conditions of stimulation, and subjects reproduced their shapes. The quality of these reproductions showed the accuracy with which these motion paths were perceived. Based on earlier results described below, in which induced motion was fully effective, we predicted that reproductions made when the motion path was given as induced motion would not be inferior to those made when real motion was given by ocular pursuit.

The other issue raised in this article is more complex. Under most circumstances, when we view motion in a lit environment, two conditions of stimulation operate simultaneously: the moving object changes its location 
relative to the stationary surround, and its motion is given subject-relatively. Both conditions of stimulation represent the same objective motion, and both would presumably result in the same perceived motion if they were presented separately. But they evoke different sensory processes that activate different perceptual channels. Configurational change is a form process, and the process that deals with ocular pursuit evaluates eye movements. Do the motions that are perceived under these conditions result only from one stimulation condition, perhaps with some subjects using configurational change and others ocular pursuit? Or do the processes that result from the different stimulation condtions come together and cooperate?

It is already known that they can cooperate. A coherent motion path is perceived when two different conditions of stimulation that represent motions of the same point in different directions result in unified motion. Such a combined motion path was first employed in the experiments of Wallach, Bacon, and Schulman (1978). When a stationary object is seen on a background of randomly placed dots that moves obliquely, the object is perceived to move obliquely in the direction opposite to the motion of the background. Here the perceived motion of the object is fully determined by the relative displacement between the object and the dot pattern. That would, however, not be the case if the background pattern consisted of endless parallel vertical lines. In such a pattern, a vertical displacement between an object and the pattern would produce no visible object-relative change, because a vertical displacement would encounter no landmarks. A vertical displacement would not be given objectrelatively, and the same would be true also of the vertical component of an oblique displacement. ${ }^{1}$ Therefore, an object-relative displacement between an object and the line pattern determines only a horizontal displacement component, and a vertical line pattern provides objectrelative displacement only in the horizontal dimension and not in the horizontal direction. This kind of object-relative displacement will tend to cause motion in some direction that has a horizontal component, that is, also motion in an oblique direction. The arrangement used by Wallach et al. (1978) yields a dimensional separation of different motion stimuli: A displacement of the line pattern causing induced motion provides for motion of an object in the horizontal dimension, whereas a simultaneous real vertical motion of that object is given only subject-relatively.

Wallach et al. (1978) presented a translucent screen with a pattern of vertical lines in reciprocating horizontal motion. A small light spot was projected on the center of the screen and was given a vertical reciprocating motion. Both motions were simple harmonic. When the motion of the spot was in phase with the motion of the screen, that is, when they always reversed motion direction at the same time, the spot was perceived to move on an oblique path. This path was the resultant of the real vertical motion of the spot and of its induced motion in the horizontal dimension, the latter a consequence of its relative displacement with respect to the line pattern. As stated, the two components of the perceived oblique path were given by different conditions of stimulation that resulted in different perceptual processes. Because the authors instructed subjects to keep their eyes on the moving spot, its vertical motion was given by ocular pursuit. The horizontal component, on the other hand, was given by the changing location of the spot relative to the lines, and the resulting perceptual process depended on the given changing configuration. Two different perceptual processes resulted: one evaluated pursuit eye movements, and the other dealt with configurational change. At some point they must have come together to produce the unified perception of oblique motion.

As already stated, two different conditions of stimulation also take place under ordinary conditions when one observes an object in motion among stationary objects. But whether, here, both resulting processes participate in causing the perceived motion is an open question. Only in an arrangement that yields dimensional separation of the different motion stimuli, as in the vertical line arrangement, can we be sure that we are dealing with simultaneous processing leading to unified motion perception.

When Wallach et al.'s (1978) subjects saw the oblique motion path that was an instance of unified perceived motion that resulted from the combination of different perceptual processes, they may have experienced the result of a perceptual event that they had never encountered before; yet they noticed nothing unusual. It was apparent that the two processes combined in an orderly fashion. The question that arose was whether this would still be so if the combined motion path was more complex. What would happen if the vertical and the horizontal motions, one given by ocular pursuit and the other by changing configuration, were combined in a different fashion?

The results of combining two simultaneous physical motions that are periodic, simple harmonic, and take place in different dimensions are well known. They can be studied, for instance, with a pendulum that is made to swing simultaneously in two planes that intersect perpendicularly, or on an oscilloscope. By changing the phase relationship between two reciprocating simple harmonic motions or their frequency ratios, or both, a family of regular motion paths-known as Lissajous figures-is obtained. The simplest of this family of motion paths is a straight line forming angles of $45^{\circ}$ with the directions of two simultaneous motions of equal extent. Changing the phase relationship between the two motions stepwise results in a number of elliptical paths or a circular path, and changing the frequency ratio between the two motions results in a great variety of motion paths.

The oblique path that resulted from the two simultaneous processes used in the experiment by Wallach et al. (1978) also can be regarded as a Lissajous figure, a psychological one, to be sure, because it does not exist in the physical environment but results from the combination of two perceptual processes of unrelated origin. The 
question that arose then was whether more complex Lissajous figures could be perceived when the phase relationship between the motion of the spot and the motion of the line pattern was altered. It was found that subjects would describe rounded motion paths that resembled circles when the line pattern changed motion direction when the motion of the spot was at the halfway point. ${ }^{2}$ It was also found that most subjects perceived with fair accuracy some of the more complex Lissajous figures that resulted from altering the frequency ratio.

The experiments to be reported here were meant to address the question of whether these "psychological" Lissajous figures (L-figures) show, in any way, that they result from the combination of different perceptual processes. For this purpose, we compared such combination L-figures with the perception of the motion paths that resulted from single stimulation. Because the two conditions of stimulation that participated in the perception of combination L-figures consisted of ocular pursuit mediating displacement of our target object in one dimension and of configurational change mediating its object-relative dispiacement in the other dimension, we arranged two conditions with which combination L-figures were to be compared. In one, real L-figure paths were shown and were mediated by ocular pursuit; in the other, both dimensions of the target displacement were given by objectrelative displacement. In the pursuit condition, a light spot was thrown from the rear onto a large translucent screen by a small tubular projector that could be moved in a variety of ways so that the light spot would move through various L-paths. The object-relative condition had to take the form of induced motion, just as was the case in our vertical-line arrangement; otherwise the objective L-path would be given not only by configurational change, but also subject-relatively. Induced motion was caused by a pattern, consisting of vertical and horizontal lines that were projected on the screen, that was made to undergo L-figure displacements with all parts of the pattern moving on parallel $\mathrm{L}$-paths. As a result, a stationary target spot on the screen was seen to move by induced motion on an L-path that was the inverse of the paths of the parts of the surrounding pattern. (The latter, on the other hand, were actually not perceived.)

Preliminary experiments showed large individual differences in subjects' abilities to reproduce the more complex L-paths. We therefore made it possible to compare the quality of $L$-path perception under different conditions, using the same subjects. We used two groups of subjects; one observed in the combination condition and in the ocular pursuit condition, and the other observed in the combination condition and in the induced-motion condition. However, by using a rating scale, we could also evaluate in absolute terms the reproductions of the various motion paths we collected. This was of value since the perception of complex motion paths when these paths were mediated by pursuit or by induced motion alone had not been studied before.

\section{METHOD}

Subjects

The two groups of 24 subjects each were undergraduate students of Swarthmore College, who were paid for their participation.

\section{Equipment}

The combination condition. The vertical line arrangement that was used for the combination condition was first described by Wallach et al. (1978). The line pattern was presented on a translucent screen $50 \mathrm{~cm}$ wide and $80 \mathrm{~cm}$ high, which was set in a metal frame. Viewed from the distance of $34.5 \mathrm{~cm}$, the lines in the pattern subtended a visual angle of $98.5^{\circ}$. The lines consisted of thin wires that were stretched between the upper and lower bars of the frame and, in the present experiment, were $1.25 \mathrm{~cm}$, or $2^{\circ}$ of visual angle, apart. A pair of rollers at each end of the base of the frame ran in retaining slots that permitted the screen to be moved back and forth horizontally and kept it in upright position. The reciprocating simple harmonic motion of the screen was provided by a scotch yoke located in the rear of the screen. The shaft of the yoke was driven by a variable (shunt) motor with a reduction gear. The reciprocating member of the yoke moved parallel to the screen and was connected to the frame of the screen by a horizontal bar. The vertically moving light spot was focused on the screen by a small projector consisting of a simple lens, a circular aperture, and small light bulb assembled inside a metal tube of $2.5-\mathrm{cm}$ diameter. The tube was attached at its center to a shaft that turned in a horizontal bearing. A lever arm that was attached to the shaft at right angles was engaged by a second scotch yoke whose reciprocating member moved in the plane in which the lever tilted. When the yoke was set in motion to push the lever arm back and forth, the projector tube tilted up and down, causing the light spot it produced on the screen to move up and down in simple harmonic motion. The shaft of the second yoke was connected to the shaft of the yoke that moved the screen by an arrangement of gears mounted on a spring-loaded lever arm. This arrangement made it possible to change the phase relationship between the yokes and, thus, between the motions of the light spot and the screen. The frequency ratio of the screen and the spot motion could be altered by changing one of the gears that connected the yokes.

The screen was illuminated by a shielded source to make the line pattern visible. The moving light spot measured $2 \mathrm{~mm}$ across. The subject sat on an adjustable chair so that his or her eyes were at the level of the midpoint of the spot's path. The vertical excursion of the spot and the horizontal excursion of the screen covered the same distance. The time it took an excursion was variable and depended on the L-figure to be presented.

The pursuit condition. For the pursuit condition, the apparatus described above was altered in the following fashion. The moving screen was removed, and a large stationary screen made of a translucent plastic sheet was put in its place. The shaft on which the projector tilted up and down was replaced by a cardanic suspension. This made it possible to change simultaneously the projector's slant angle in the horizontal plane and its slant in the vertical dimension. The control of its vertical slant remained the same as before, but a new linkage system connected the horizontal slant with the yoke that previously had caused the horizontal motion of the screen. Thus, one yoke now controlled the projector's slant in the horizontal plane, and the other controlled its slant in the vertical plane. This arrangement made it possible to have the projected light spot trace out various real L-figures on the screen. ${ }^{3}$

To check the accuracy with which the projected spot moved through the L-path, tracing paper was attached to the screen and used in the following manner. The experimenter repeatedly gave the primary shaft of the motor that moved the two yokes a few turns by hand, each time advancing the projected spot a small step on 
its path. Each new location of the spot was marked on the tracing paper until the whole L-path was traced out. In this fashion, tracings of all $10 \mathrm{~L}$-paths that were used in the experiment were obtained.

The induced-motion condition. The apparatus was further modified for the induced-motion condition, in which a large pattern moved in real $\mathrm{L}$-figure fashion to induce $\mathrm{L}$-figure motion in a stationary light spot. A small mirror was mounted on the front end of the projector, whose direction underwent the L-figure motion in the pursuit condition. When the projector went through its motions, the changing slant angles of the mirror imparted L-figure motion to a light beam it reflected. This light beam carried the inducing pattern, which consisted of equidistant vertical and horizontal lines, onto the screen. A slide with this pattern was inserted into a lantern equipped with a wide-angle lens. The lantern beam was initially parallel to the screen. A beam splitter, placed between the moving mirror and the screen, reflected a proportion of the lantern beam onto the moving mirror, which, in turn, reflected it forward toward the screen, where a fraction of it came into focus after passing through the beam splitter. To make the excursions of the L-figure motions of the projected pattern equal to the excursions of the projected light spot in the pursuit condition, the slanting motions of the projector, and hence those of the moving mirror, were diminished by a factor of 2 . A separate stationary lantern was used to project the immobile light spot so that it was centered in the projected pattern.

The subject reproduced an observed motion path by shaping a rope that was lying on a white table top. This rope consisted of a flexible tube made of blue bias tape and filled with fine bird shot to make it heavy. Its diameter was $1.5 \mathrm{~cm}$ and its length was $216 \mathrm{~cm}$. A 35-mm camera equipped with a yellow filter was mounted above the table. The camera was focused on the blue rope, and a black and white picture was taken of each reproduction and eventually made into a slide.

\section{Procedure}

Five different Lissajous paths were observed in two orientations under the following three presentation conditions: (1) PursuitThe spot moved on a dark screen, and the subject was instructed to track it. (2) Induced motion-A stationary spot was seen on a pattern of crossing lines that moved; the subject was instructed to keep looking at the spot. (3) Combination-A spot moved vertically and a pattern of vertical lines moved horizontally. The subject was instructed to track the spot.

Figure 1 shows the five L-paths in the two orientations in which each was used. Table 1 lists these L-paths in the order in which they appear in Figure 1 by code names that connote their shapes. The frequency ratios and phase relations that define these paths are given, as is the time it took for one completion of each motion path. In the case of the three open paths, motions reversed direction at the paths' ends, so that each path was alternately traversed in opposite directions.

Two versions in different orientations were needed for each path, since each subject reproduced the same path under two presentation conditions. The 24 subjects of Group 1 observed in the pursuit condition and in the combination condition; the 24 subjects of Group 2 observed in the induced-motion condition and in the combination condition. The subjects came for two sessions at least 1 week apart. In one session, they were presented with the combination condition; in the other session, they were presented with either the pursuit condition (Group 1) or the induced-motion condition (Group 2). Also, in one session, they were presented with the paths listed after $A$ in Table 1; in the other, they were presented with those listed after $B$. The combination of these variables was properly designed. The L-paths were presented in two counterbalanced sequences, in either ascending or descending order of complexity. For a particular subject, the order was the same in the two experimental sessions.

Both the horizontal reciprocating motion of the line pattern and the vertical excursion of the spot in the combination condition, the horizontal and the vertial motion components that resulted in the various L-paths in the pursuit condition, and the motion components of the pattern of crossing lines in the induced-motion condition each amounted to $8 \mathrm{~cm}$. The subject, with his or her head in a chinrest, observed the displays from a distance of $34.5 \mathrm{~cm}$, so that $8 \mathrm{~cm}$ on the display amounted to $13^{\circ}$ of visual angle. In every exposure of the motion display, the motion path was completed twice without a pause; the experimenter, however, informed the subject when the first motion path was completed. For the two closed paths, presentations always started at the same point; for the open paths, the presentation started at a point at which the motion direction reversed. The subject was informed in advance of each exposure as to whether the second tracing of the path was in the same direction as the first or in the opposite direction.

As soon as a path had been traced twice, a table beside the subject was illuminated and the subject had to reproduce the shape of the motion path with the heavy blue rope. Two pieces of white cardboard were available to cover those ends of the rope that were not representing the motion path. The subject was advised beforehand that the sizes of the reproductions were not important but that the shape and the orientation would have to be as accurate as possible: "When these figures are analyzed, they are not simply judged right
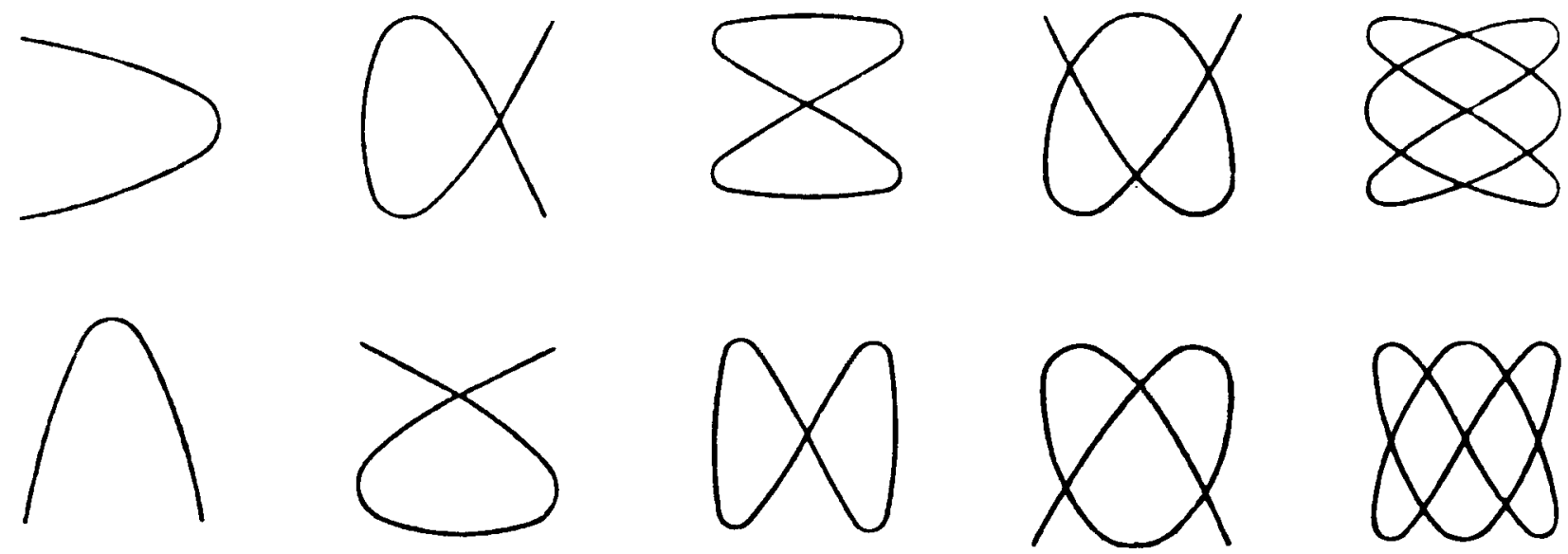

Figure 1. The five Lissajous paths in the two orientations in which each was used. 
Table 1

Defining Properties of Five Lissajous Paths in Two Orientations, $A$ and $B$ (Frequency Ratios Horizontal to Vertical and Phase Angle), and Durations of Single Paths

\begin{tabular}{|c|c|c|c|c|c|}
\hline \multirow{2}{*}{$\begin{array}{l}\text { L-Path by } \\
\text { Code Name }\end{array}$} & \multicolumn{2}{|c|}{ Frequency Ratio } & \multicolumn{2}{|c|}{ Phase Angle } & \multirow{2}{*}{$\begin{array}{l}\text { Duration } \\
\text { (in Sec) }\end{array}$} \\
\hline & A & B & A & B & \\
\hline Bow & $2: 1$ & $1: 2$ & $0^{\circ}$ & $0^{\circ}$ & 6 \\
\hline Alpha & $2: 3$ & $3: 2$ & $0^{\circ}$ & $0^{\circ}$ & 9 \\
\hline Eight & $4: 2$ & $2: 4$ & $90^{\circ}$ & $90^{\circ}$ & 12 \\
\hline Pretzel & $3: 4$ & $4: 3$ & $0^{\circ}$ & $180^{\circ}$ & 9 \\
\hline Complex & $6: 4$ & $4: 6$ & $90^{\circ}$ & $90^{\circ}$ & 18 \\
\hline
\end{tabular}

or wrong. Rather, comparisons will be made between different figures, and small differences between them may turn out to be important." After the subject had formed the figure, he or she observed another exposure of the motion path and then checked the reproduction. The subject had the option of requesting as many further exposures as he or she felt were needed. When the subject was satisfied with his or her reproduction, it was photographed after the experimenter had placed a card with an identifying code number next to it. At the beginning of the experiment, the subject was instructed in the use of the rope and the cardboard pieces. The experimenter carefully formed a circle out of part of the rope and covered, with the cardboards, the rope ends that were in excess. Each group of 24 subjects made 240 reproductions, 120 for the combination condition and 120 for either the pursuit or the inducedmotion condition.

\section{RESULTS}

In spite of large differences in the subjects' abilities to make reproductions of the various motion paths, most of the reproductions showed shapes that resembled the shown Lissajous paths or those that the displays were meant to represent. An exception was the reproductions of the "complex" path; they differed greatly from each other. There were a few almost perfect reproductions and many completely inept attempts that bore no resemblance to even part of this path. Inasmuch as completion of this path took $18 \mathrm{sec}$, it is likely that short-term memory was the limiting factor influencing reproduction of this path. Almost all reproductions of the simpler paths, the "bow" and the "alpha," were very accurate, and no differences connected with the presentation conditions were noticeable at all.

Several methods of evaluating the reproductions were used. The results of two of them will be reported in de-

Table 2

Mean Number of Times a Reproduction from One Presentation Condition was Judged to be More Like the Tracing Than was the Reproduction Made by the Same Subject in the Other Presentation Condition, and Mean Number of Ties

\begin{tabular}{lccccccc}
\hline & \multicolumn{3}{c}{ Group 1 } & \multicolumn{3}{c}{ Group 2 } \\
\cline { 2 - 5 } \multicolumn{1}{c}{ Path } & $\begin{array}{c}\text { Combi- } \\
\text { nation }\end{array}$ & Pursuit & Ties & $\begin{array}{c}\text { Combi- } \\
\text { nation }\end{array}$ & $\begin{array}{c}\text { Induced } \\
\text { Motion }\end{array}$ & Ties \\
\hline Bow & 7.25 & 5.25 & 11.5 & 5.75 & 6.0 & 12.25 \\
Alpha & 8.75 & 5.5 & 9.75 & 6.75 & 8.0 & 9.25 \\
Eight & 7.0 & 8.25 & 8.75 & 6.25 & 8.75 & 9.0 \\
Pretzel & 5.75 & 11.5 & 6.75 & 3.0 & 13.75 & 7.25 \\
Complex & 6.75 & 6.0 & 11.25 & 6.0 & 7.75 & 10.25 \\
Averages & 7.1 & 7.3 & 9.6 & 5.55 & 8.85 & 9.6 \\
\hline
\end{tabular}

tail. The method of paired comparison was designed to find out whether the reproductions of the combination Lfigures reflected the greater complexity of the underlying processes and were poorer than the reproductions made when only one stimulus condition carried the motion information, that is, when the L-paths were really shown and were given by ocular pursuit (Group 1) or when they were given as induced motion caused by a pattern undergoing real Lissajous motion (Group 2).

\section{Paired Comparison}

A rater was shown two slides simultaneously, one showing the reproduction of a combination path and the other the reproduction of one of the stimulus paths of the same L-shape produced by the same subject. (Two identical Kodak slide projectors were available.) The rater was also shown a tracing made when a single light spot went through the same L-path. The rater had to compare the two reproductions with the tracing and tell which of the reproductions appeared to be more similar to the tracing. Four raters, paid undergraduates, performed this task. With each rater comparing the reproductions of five Lpaths made by 24 subjects, for a total of 120 , a total of 480 comparisons were made for the reproductions of each group of subjects.

For each pair of L-paths, a count was made of how many reproductions made by different subjects under one presentation condition were judged by a rater to be more like the tracing than the reproduction made under the other presentation conditions. These preference counts for each version of an L-path made by the four raters were averaged. These means are listed in Table 2.

Except for the L-path "pretzel," the mean preference counts for the presentation condition under comparison were not meaningfully different from each other. In the case of the "pretzel" path, the reproductions made under the single-stimulation conditions had larger mean preference counts than those made in connection with the combination condition. This difference was significant in the case of Group 2, whose reproductions for the induced motion and the combination conditions were compared. A McNemar test showed that the number of judgments favoring the induced-motion condition was significantly greater than those favoring the combination condition $\left[\chi^{2}(1)=5.68, p<.01\right]$. It seems possible that inaccurate motion perception would be manifest only with stimuli of intermediate complexity such as the "pretzel" path. 
Table 2 shows that the mean number of ties, averaging $40 \%$, were high throughout. An inspection of the reproductions showed that the reason for the ties connected with the "bow" and the "alpha" paths resulted from the high number of completely correct reproductions, whereas a large number of reproductions of the "complex" path were so poor that it was impossible to rate one as better than the other. We have mentioned earlier that few subjects could handle the "complex"' Lpath. In the case of Group 1, the average preference counts show no disadvantage of the combination condition, and the difference for Group 2 is due to the difference for the "pretzel" path.

The results of the paired comparisons do not show much of an adverse effect of the combination of different processes on the perceived motion paths. Paired comparison of the reproductions of as many as 24 subjects is a sensitive test, and it is difficult to know how much of a difference in the quality of the reproductions the results of the judgments connected with the "pretzel" path may reflect. However, it is possible that a difference in the quality of the reproductions occurs just in such paths of intermediary difficulty. A second method of evaluating the reproductions was meant to deal with this issue. The quality of individual reproductions was rated on a scale of 1 to 5 . Because the reproductions for the "bow" and the "alpha" L-paths were mostly correct and those of the "complex" L-path were too poor, individual ratings were obtained for only the "eight" and for the "pretzel" paths.

\section{Rating Scale}

The raters viewed one reproduction at a time together with a tracing of the L-path in question. They were instructed to evaluate the resemblance of the projected figure to the tracing, with a scale rating of 1 to be given to reproductions that were most similar to the tracing and a rating of 5 to be given to those that were most dissimilar. In the case of each L-path, a rater was made acquainted with the variations in the reproductions. The 48 reproductions of an $L$-path made by the subjects of one of the groups under the two presentation conditions were arranged in random order and were first presented one at a time. This acquainted the rater with the range of variability. Then each of the reproductions of that set was presented again for the actual assignment of a rating. All reproductions were shown in the $B$ orientation: The reproductions of the vertical "eight" were turned by $90^{\circ}$ so that the raters saw each "eight" on its side. The open ends of all "pretzels" pointed downward.

Here, too, four raters were employed. Their ratings were compared to assess interrater reliability. The index used was the proportion of reproductions on which the ratings assigned by three raters differed by not more than 1 point. Interrater reliability was found to be above .9 in all sets.

Resemblance ratings were obtained for each L-path by averaging the ratings for individual reproductions made for a representation condition by the four raters. The means of these resemblance ratings are listed in Table 3 . Because the variability in the ratings for the "eight" paths in the upright position was higher, separate means were computed for the resemblance ratings for the two orientations of the "eight" paths.

In five of the six comparisons that can be made between the mean ratings in the combination conditions and those in the single-stimulus conditions, the mean ratings were lower, that is, better, in the latter conditions, and in one case the difference was significant. All differences, however, were quite small and are not indicative of a serious flaw in the perception of the motion paths in the combination conditions. These small differences may have resulted because two conditions of stimulation, rather than a single one, operate in the combination condition, each with its own source for error. They need not reflect an adverse effect of the combination process in which two stimulus processes must participate before a single motion and a motion path are perceived.

Another comparison shows that the quality of the reproductions made for the induced-motion presentations was as good as the quality of the reproductions of the physical L-paths when they were given by ocular pursuit. This finding is also supported by the paired comparisons listed in Table 2. For every L-path, the preference count is slightly higher for the induced-motion presentation than for the process combination paths; this is not the case in the paired comparisons between the combination paths and the physical L-paths. Configurational change is a fully effective stimulation condition even when it manifests itself as induced motion.

\section{DISCUSSION}

Our investigation of the perception of motion paths had two results. When the perceived motions were the out-

Table 3

Mean Ratings on a Scale of 1 (Best) to 5 (Worst) of Resemblance of Reproductions to Tracings and $t$ Scores of Differences Between Compared Reproductions

\begin{tabular}{|c|c|c|c|c|c|c|}
\hline \multirow[b]{2}{*}{ Path } & \multicolumn{3}{|c|}{ Group 1} & \multicolumn{3}{|c|}{ Group 2} \\
\hline & Combination & Pursuit & $t$ & Combination & $\begin{array}{l}\text { Induced } \\
\text { Motion }\end{array}$ & $t$ \\
\hline Vertical Eight & 3.29 & 3.46 & .32 & 3.0 & 2.79 & .54 \\
\hline Horizontal Eight & 2.83 & 2.17 & 1.15 & 2.75 & 2.42 & .80 \\
\hline Pretzel & 3.12 & 2.85 & 1.32 & 3.42 & 2.88 & $3.09^{*}$ \\
\hline Averages & 3.08 & 2.83 & & 3.06 & 2.70 & \\
\hline
\end{tabular}

$* d f=23, p<.01$. 
come of a combination of two motion processes that resulted from different stimulations, the perceived motion paths were not indicative of a serious impairment of the underlying motion perception-in spite of the fact that the combination of different motion processes in different dimensions was undoubtedly a new event in our subjects' perceptual functioning. This suggests that the two processes cooperate when, under ordinary conditions, they are both simultaneously evoked by stimulations that mediate the same objective motion and result in the same perceived motion.

The finding that perception of complex motion paths that is based on induced motion is of the same quality as motion path perception based on viewing real motion paths throws new light on induced motions. It shows that motion perceived on the basis of induction can be equal to motion perception that is caused by real motion. This raises the question of why, in so many instances, induced motion is diminished (e.g., Rock, Auster, Schiffman, \& Wheeler, 1980) or even fails altogether. We believe that this discrepancy is due to the fact that in our experiment parts of the inducing pattern are at all times closer to the target and that the inducing pattern is thus a better representation of the visual environment in relation to which moving objects are displaced under ordinary conditions and with which they form configural changes. By contrast, in numerous experiments on induced motion, the visual environment is represented by a mere outline figure. Such outlines are poor representations of the realistic environments that normally participate in the object-relative displacements that occur when objects move.

\section{REFERENCES}

Becklen, R., \& Wallach, H. (1985). How does speed change affect induced motion? Perception \& Psychophysics, 37, 231-236.
Duncker, K. (1929). Über induzierte Bewegung. Psychologische Forschung, 12, 180-259.

Post, R. B. (1986). Induced motion considered as a visually induced oculogyral illusion. Perception, 15, 131-138.

REGAN, D. (1986). Visual processing of four kinds of relative motion. Vision Research, 26, 127-145.

Rock, I. (1983). The logic of perception. Cambridge, MA: MIT Press.

Rock, I., Auster, M., SchiffMan, M., \& WheEler, D. (1980). Induced movement based on subtraction of motion from the inducing object. Journal of Experimental Psychology: Human Perception \& Performance, 6, 391-403.

WaLlaCH, H. (1976). On perception. New York: Quadrangle/The New York Times Book Co.

Wallach, H., Bacon, J., \& Schulman, P. (1978). Adaptation in motion perception: Alteration of induced motion. Perception \& Psychophysics, 24, 509-514.

WALLACH, H., \& BECKLEN, R. (1983). An effect of speed on induced motion. Perception \& Psychophysics, 34, 237-242.

\section{NOTES}

1. As a corollary, endless straight lines cannot transmit a definite motion direction. Nevertheless, a definite motion direction is perceived when such lines are displaced; they are perceived to move perpendicularly to their extension, and that is the reason why the line pattern appears to move horizontally in the arrangement in Wallach et al. (1978). But, as stimuli involved in object-relative displacement, the perceived motion direction of the lines is irrelevant. Why straight lines tend to move perpendicularly to their extensions is discussed in Wallach (1976) on pages 202-203.

2. Such an arrangement has been used by Wallach and Becklen (1983) and by Becklen and Wallach (1985) to measure induced motion. Instead of estimates of the slope angle of the oblique motion path used by Wallach et al. (1978), the ratio of estimates of width and height of perceived elliptic motion paths served as measurements of induced motion.

3. We are grateful to Crispin Miller for designing this arrangement.

(Manuscript received May 29, 1987;

revision accepted for publication September 25,1987 .) 